\title{
EDITORIAL
}

\section{Alveolar epithelial type II cells from embryonic stem cells: knights in shining armour?}

\author{
H. Fehrenbach
}

$\mathbf{T}$ oday, the pulmonary epithelium is generally accepted to possess unique properties that go far beyond the role as a mere physical barrier protecting an organism's interior against the detrimental effects exerted by airborne environmental challenges, such as gases, particles, allergens or microorganisms. The specific tasks respiratory epithelial cells have to fulfil differ along the bronchiotracheal tract down to the alveoli $[1,2]$. Accordingly, there is a continuous change in the cellular composition of the epithelium along the airway tract reflecting remarkable structural and functional differentiation. Besides long-known roles of the epithelium, such as mucociliary clearance, lung fluid balance and surfactant homeostasis, which assure the removal of particles and cells, the avoidance of oedema and the proper adjustment of surface tension, respectively, are additional important functions that have been identified in recent years related to the regulation of innate and adaptive immune responses [3-6].

In order to guarantee that these functions, which are critical for the proper function of the lungs, can be maintained continuously, mechanisms of epithelial repair and regeneration involving a plethora of mediators and pathways have evolved [7]. Regionspecific epithelial "task forces", i.e. local progenitor cells including alveolar epithelial type II cells (AECII), or perhaps only a specific subset of these [8], are "defined" to accomplish epithelial repair and regeneration in the different regions along the respiratory tract [9-11]. Until a recent study reported the identification of a distinct population of adult human lung stem cells that were suggested to exhibit characteristics of pluripotent stem cells [12], a report that provoked vigorous debate [11, 13, 14], the classical stem cell hierarchy typical for other organs appeared to be absent in the lung [15]. We can certainly expect that the near future will provide us with exciting insights into this hot topic.

Focussing on the lung parenchyma, AECII are considered the defenders of the alveolus because they are crucial for the protection of the alveolar microenvironment in many ways [2]. Even if AECII fail as defenders in warding off an attack, they appear to be capable of initiating repair processes, maintaining or even restoring the complex alveolar architecture [16]. Consequently, various experimental approaches were followed to strengthen the protective and regenerative capacities of AECII, for example, by introducing exogenous epithelial growth factors implicated in regular repair processes [7], such as keratinocyte growth factor [17] and hepatocyte growth factor [18], or stimulating their endogenous synthesis.

CORRESPONDENCE: H. Fehrenbach, Section of Experimental Pneumology, Research Center Borstel, Parkallee 1, Borstel, D-23845, Germany. E-mail: hfehrenbach@fz-borstel.de
In turn, this concept of the role of AECII implies that severe damage to or loss of AECII may result in a considerable vulnerability of the alveolus and the impairment of alveolar repair. Consequently, direct damage to AECII and disturbance of repair processes may be implicated in the pathogenesis of various pulmonary disorders, such as acute lung injury [19, 20], chronic obstructive pulmonary disease/emphysema [21, 22] and lung fibrosis [23-25]. Particularly with regard to idiopathic pulmonary fibrosis, convincing evidence has been gathered over recent years to support the notion that the fibrotic response is largely driven by abnormally activated AECII, as comprehensively reviewed previously $[26,27]$. Of course, in order to gain a full understanding of the role of AECII in lung fibrosis, their interactions with extracellular matrix components and interstitial (mesenchymal) cells have to be taken into account [28].

In this context of AECII being lost or abnormally activated, therapeutic approaches aiming to stimulate AECII may not only fail (due to the missing target) but, even worse, might potentiate the pathogenetic process. The study of SPITALIERI et al. [29] published in this issue of the European Respiratory Journal demonstrates a potential loophole: the in vitro differentiation of AECII from human embryonic stem cells (HUES) for use in a cell therapeutic approach. The authors used various methodological approaches to broadly analyse the molecular and functional phenotype of AECII differentiated from HUES. Therapeutic application of such cells in humans will require considerable expansion of HUES or the AECII derived from them in order to obtain adequate cell numbers. On the one hand, expansion of HUES is not a trivial task and many aspects have to be taken into account, as described recently [30]. On the other hand, the expansion of AECII differentiated from HUES requires that the differentiated phenotype is maintained, which in differentiated AECII isolated from the lung and cultured in vitro, again requires special efforts [31]. Such technical problems may be resolved in the future if adequately addressed. Most notably, SPITALIERI et al. [29] tested the AECII differentiated from HUES in an in vivo animal model of pulmonary fibrosis for their therapeutic potential. Unlike many other animal studies in this field employing a bleomycin model of fibrosis, which has clear limitations [32], the authors used a silica model, which has the advantage that there is a persistent fibrotic stimulus due to the repetitive instillations as well as the limited clearance of the particles [33]. Moreover, cell therapy was initiated after 15 days of silica treatment, which indicates that the setting is close to a realistic therapeutic situation, with the disease already being established before the intervention was initiated. Although the histopathological findings, which for a stereologist such as myself, could 
have been more rigorous, clearly indicate that the progression of the disease was inhibited by AECII differentiated from HUES, in contrast to a control therapy using human fibroblasts, a therapeutic effect in the sense of achieving a near-normal lung architecture remains to be demonstrated. Furthermore, the authors have shown nicely that various cytokines (in particular, transforming growth factor- $\beta$, the major player in the pathogenetic process) and collagen 1 gene expression were significantly inhibited by their therapeutic approach. Data on the effects on the respective protein levels, however, would have further improved the impact of this study. Additional experiments are warranted in the future to reveal the precise mechanisms of this therapeutic approach. Specifically, knowledge of the potential interactions of AECII introduced into the lung with resident mesenchymal cells will probably enhance our understanding of the relevance of this experimental therapeutic approach. In particular, it might be interesting to learn if and how AECII differentiated from HUES and introduced into a fibrotic lung escape from being abnormally activated like the resident AECII. Hence, the study of SPITALIERI et al. [29] has opened a new avenue along which AECII differentiated from HUES may come along as a knight in shining armour to help us to combat a still lifethreatening and poorly manageable lung disease. Combining this approach with genetic manipulation may even help to further strengthen the AECII's armour [34]. However, history and myths tell us that seemingly invincible knights bear armour that, in most cases, revealed a specific point of weakness, and it is the task of science to identify such points of weakness in future studies in order to improve our therapeutic armamentarium against lifethreatening diseases, such as pulmonary fibrosis.

\section{STATEMENT OF INTEREST}

None declared.

\section{REFERENCES}

1 Crystal RG, Randell SH, Engelhardt JF, et al. Airway epithelial cells: current concepts and challenges. Proc Am Thorac Soc 2008; 5: 772-777.

2 Mason RJ. Biology of alveolar type II cells. Respirology 2006; 11: Suppl., S12-S15.

3 Wright JR. Immunoregulatory functions of surfactant proteins. Nat Rev Immunol 2005; 5: 58-68.

4 Bals R, Hiemstra PS. Innate immunity in the lung: how epithelial cells fight against respiratory pathogens. Eur Respir J 2004; 23: 327-333.

5 Bulek K, Swaidani S, Aronica M, et al. Epithelium: the interplay between innate and Th2 immunity. Immunol Cell Biol 2010; 88: 257-268.

6 Vareille M, Kieninger E, Edwards MR, et al. The airway epithelium: soldier in the fight against respiratory viruses. Clin Microbiol Rev 2011; 24: 210-229.

7 Crosby LM, Waters CM. Epithelial repair mechanisms in the lung. AJP Lung Cell Mol Physiol 2010; 298: L715-L731.

8 Chapman HA, Li X, Alexander JP, et al. Integrin $\alpha 6 \beta 4$ identifies an adult distal lung epithelial population with regenerative potential in mice. J Clin Invest 2011; 121: 2855-2862.

9 Kotton DN, Fine A. Lung stem cells. Cell Tiss Res 2008; 331: 145156.

10 Rock JR, Hogan BLM. Epithelial progenitor cells in lung development, maintenance, repair, and disease. Annu Rev Cell Dev Biol 2011; 27: 493-512.
11 Anversa P, Kajstura J, Leri A, et al. Tissue-specific adult stem cells in the human lung. Nat Med 2011; 17: 1038-1039.

12 Kajstura J, Rota M, Hall SR, et al. Evidence for human lung stem cells. N Engl J Med 2011; 364: 1795-1806.

13 Stripp B, Hogan B, Thannickal VJ. Lung stem cells: looking beyond the hype. Nat Med 2011; 17: 788-789.

14 Chapman HA. Toward lung regeneration. N Engl J Med 2011; 364: 1867-1868.

15 Rawlins EL, Hogan BLM. Epithelial stem cells of the lung: privileged few or opportunities for many? Development 2006; 133: 2455-2465.

16 Yildirim AO, Muyal V, John G, et al. Palifermin induces alveolar maintenance programs in emphysematous mice. Am J Respir Crit Care Med 2010; 181: 705-717.

17 Sakamoto S, Yazawa T, Baba Y, et al. Keratinocyte growth factor gene transduction ameliorates pulmonary fibrosis induced by bleomycin in mice. Am J Respir Cell Mol Biol 2011; 45: 489-497.

18 Gazdhar A, Fachinger P, van Leer C, et al. Gene transfer of hepatocyte growth factor by electroporation reduces bleomycin-induced lung fibrosis. AJP Lung Cell Mol Physiol 2007; 292: L529-L536.

19 Miyake $\mathrm{Y}$, Kaise H, Isono K-I, et al. Protective role of macrophages in noninflammatory lung injury caused by selective ablation of alveolar epithelial type II Cells. J Immunol 2007; 178: 5001-5009.

20 McElroy MC, Kasper M. The use of alveolar epithelial type I cellselective markers to investigate lung injury and repair. Eur Respir J 2004; 24: 664-673.

21 Petrache I, Natarajan V, Zhen L, et al. Ceramide upregulation causes pulmonary cell apoptosis and emphysema-like disease in mice. Nat Med 2005; 11: 491-498.

22 Rennard SI, Wachenfeldt K. Rationale and emerging approaches for targeting lung repair and regeneration in the treatment of chronic obstructive pulmonary disease. Proc Am Thorac Soc 2011; 8: 368-375.

23 Kasper M, Haroske G. Alterations in the alveolar epithelium after injury leading to pulmonary fibrosis. Histol Histopathol 1996; 11: $463-483$

24 Sisson TH, Mendez M, Choi K, et al. Targeted injury of type II alveolar epithelial cells induces pulmonary fibrosis. Am J Respir Crit Care Med 2010; 181: 254-263.

25 Drakopanagiotakis F, Xifteri A, Polychronopoulos V, et al. Apoptosis in lung injury and fibrosis. Eur Respir J 2008; 32: 1631-1638.

$26 \mathrm{du}$ Bois RM. Strategies for treating idiopathic pulmonary fibrosis. Nat Rev Drug Discov 2010; 9: 129-140.

27 King TE, Pardo A, Selman M. Idiopathic pulmonary fibrosis. Lancet 2011; [Epub ahead of print DOI: 10.1016/S0140-6736(11)60052-4].

28 Chapman HA. Epithelial-mesenchymal interactions in pulmonary fibrosis. Annu Rev Physiol 2011; 73: 413-435.

29 Spitalieri P, Quitadamo MC, Orlandi A, et al. Rescue of murine silica-induced lung injury and fibrosis by human embryonic stem cells. Eur Respir J 2012; 39: 446-457.

30 Zweigerdt R, Olmer R, Singh H, et al. Scalable expansion of human pluripotent stem cells in suspension culture. Nat Protoc 2011; 6: 689-700.

31 Borok Z, Crandall ED. More life for a "terminal" cell. AJP Lung Cell Mol Physiol 2009; 297: L1042-L1044.

32 Moeller A, Ask K, Warburton D, et al. The bleomycin animal model: a useful tool to investigate treatment options for idiopathic pulmonary fibrosis? Int J Biochem Cell Biol 2008; 40: 362-382.

33 Degryse AL, Lawson WE. Progress toward improving animal models for idiopathic pulmonary fibrosis. Am J Med Sci 2011; 341: 444-449.

34 Leblond A-L, Naud P, Forest V, et al. Developing cell therapy techniques for respiratory disease: intratracheal delivery of genetically engineered stem cells in a murine model of airway injury. Hum Gene Ther 2009; 20: 1329-1343. 